\title{
Novel antibody blocks thrombus formation
}

\begin{abstract}
Although agents that target platelets have been clinically successful in the treatment and prevention of cardiovascular disease, their safety and efficacy remain limited. Now, writing in the Journal of Clinical Investigation, Takayama and colleagues describe the development of a novel antiplatelet therapy, based on a human collagen receptor autoantibody.

Vascular damage results in the exposure of subendothelial matrix proteins such as collagen, which interact with cellular collagen receptors to promote platelet adhesion and activation, leading to thrombus formation. One such receptor, glycoprotein VI (GPVI), plays a crucial role in this platelet response and has therefore become a promising target for antiplatelet therapies.
\end{abstract}

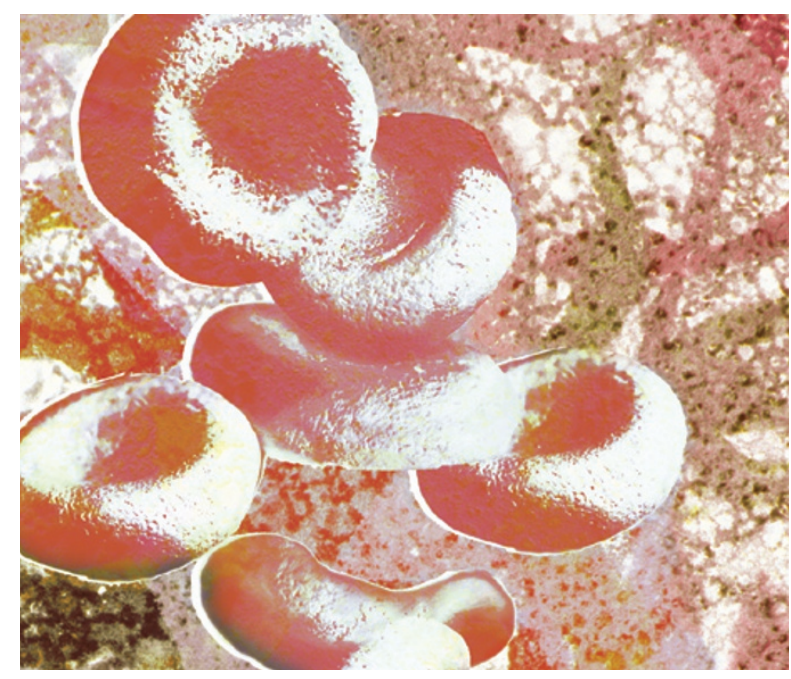

Over 20 years ago, a patient with platelet GPVI deficiency and unresponsiveness to collagen, owing to the development of autoantibodies against GPVI, was identified. Today, this patient still has platelets that lack GPVI, without thrombocytopaenia or any signs of bleeding. Therefore, with the ultimate aim of developing clinically useful monoclonal antibodies (mAbs) that target human GPVI (hGPVI), the authors set out to characterize these autoantibodies.

Purification of autoantibodies from the patient's plasma and examination of cross-reactivities with mouse anti-GPVI mAbs for binding to hGPVI identified two representative $\mathrm{mAbs}, \mathrm{mF} 1201$ and $\mathrm{mF} 1232$, for further study. In vitro, mF1232 inhibited human collagen-induced platelet aggregation in a dose-dependent manner, whereas mF1201 induced platelet activation and cell-surface GPVI shedding. When injected into the cynomolgus monkey, both antibodies caused GPVI immunodepletion, accompanied by an unresponsiveness of platelets to collagen. However, mF1201 also exerted significant thrombocytopaenia. These initial studies therefore suggested that mF1232 was the more promising therapeutic agent. Moreover, $\mathrm{mF} 1232$ also recognizes a novel epitope on loop 9 of immunoglobulin D2 of hGPVI.

To investigate the mechanism of action of mF1232, the authors constructed a human-mouse chimera of mF1232 (cF1232), which, importantly, exerted identical actions to that of mF1232 in the studies reported so far. Labelling of cF1232 with a fluorescent endocytosis probe (CypHer5E) identified a rapid increase in GPVI levels in circulating platelets following intravenous injection into monkeys. Surface GPVI levels were also dramatically reduced. Antibody binding and uptake was shown to be dependent upon the surface expression of GPVI. That is, injection of unlabelled cF1232 (to immunodeplete surface GPVI) before CypHer5E-labelled cF1232 decreased fluorescence. Such loss of surface GPVI expression and endocytosis of cF1232 into platelets was reproduced in vitro, when monkey platelets were treated with PGE1 - a potent elevator of intracellular cyclic AMP. The authors therefore concluded that in vivo, cF1232 acts by inducing cAMP-dependent endocytosis of GPVI.

Finally, subcutaneous injection of a single dose of cF1232 into monkeys decreased GPVI surface expression for up to 7-10 days, in conjunction with unresponsiveness to collagen and without thrombocytopaenia. These results emphasize the potential clinical application of $\mathrm{cF} 1232$ as a novel antiplatelet therapy.

\section{Sarah Crunkhorn}

ORIGINAL RESEARCH PAPER Takayama, H. et al. A novel antiplatelet antibody therapy that induces cAMP-dependent endocytosis of the $\mathrm{GPVI} / \mathrm{Fc}$ receptor $\gamma$-chain complex. J. Clin. Invest. 118, 1785-1795 (2008) 